\title{
Artspezifische Merkmale der Faeces von vier dominierenden Copepodenarten der Kieler Bucht
}

\author{
P. MARTENS \\ Biologische Anstalt Helgoland (Littoralstation); List/Sylt \\ Bundesrepublik Deutschland
}

\begin{abstract}
Species-characteristics of the fecal pellets of four dominating copepod species of Kiel Bight. Mean length, width and shape of the fecal pellets of the four planktonic copepod species, Acartia bifilosa, Centropages hamatus, Oithona similis and Pseudocalanus elongatus, were studied. Mean volumes of the fecal pellets of the different species vary between $13 \times 10^{3} \mu \mathrm{m}^{3}$ (males and females of Oithona similis) and $348 \times 10^{3} \mu \mathrm{m}^{3}$ (females of Acartia bifilosa). The dimensions of the fecal pellets of the four species examined reveal significant differences in at least one of the criteria studied. In Acartia bifilosa and Centropages bamatus even the length and width of the fecal pellets of the two sexes are different. With respect to the characteristics described, a comparison made between fecal pellets found in cultures with those obtained from sediment of the Baltic Sea (Kiel Bight) has shown that the latter may be attributed to the copepod species under consideration.
\end{abstract}

\section{EINLEITUNG}

Eine bedeutende Rolle im Stoffkreislauf innerhalb eines marinen Nahrungsnetzes spielt die Formung partikulärer Substanz in Gestalt von Faeces, besonders durch planktische Copepoden. Als dominierende Formen bilden sie in der Kieler Bucht durchschnittlich $70 \%$ des Zooplanktons (HnlebrandT, 1972). Copepoden geben ihre Faeces nicht als amorphe Masse ab, sondern als kleine Körper bestimmter Form und Größe, die von einer peritrophischen Membran umgeben sind (FonsTER, 1953; Gould, 1957). Diese führt dazu, daß die Kotballen eine sehr viel höhere Sinkgeschwindigkeit haben als tote Algen oder auch Zooplankter, da ihr Formwiderstand geringer ist. So liegen die Sinkgeschwindigkeiten der Faeces planktischer Crustaceen zwischen $43 \mathrm{~m}$ und $376 \mathrm{~m}$ pro Tag (Osterberg et al., 1963; SMAyda, 1969).

Phyto- und Zooplankter werden bei der Sedimentation in größeren Wassertiefen durch bakterielle und chemische Zersetzung weitgehend abgebaut, so daß nur energiearme, schwer abbaubare Verbindungen den Boden erreichen. Die Faeces dagegen mit ihrem hohen Anteil an organischer Substanz (bis 72\%) (NEwell, 1965; JoHannes \& SAtomi, 1966) werden auf Grund der stabilen Hüllmembran (Moore, 1931) kaum zersetzt. Dies führt zu einem schnellen Transport hochwertiger organischer und anorganischer Substanzen aus produktionsreichen Wasserschichten in die Tiefe (OsTER- 
BERg et al., 1963; Schrader, 1971), wo sie für das Benthos eine wichtige Nahrungsund Nährstoffquelle bilden.

In dieser Arbeit soll untersucht werden, ob den Faeces der verschiedenen Copepodenarten der Kieler Bucht artspezifische Merkmale eigen sind und ob bei deren Kenntnis eine Analyse des sedimentierten Sestons Rückschlüsse auf Vorgänge innerhalb der Zooplanktonpopulation der Wassersäule zuläßt.

\section{MATERIAL UND METHODE}

Folgende planktischen Copepodenarten wurden untersucht: Acartia bifilosa, Centropages hamatus, Oithona similis und Pseudocalanus elongatus. Sie bilden im Jahresdurchschnitt den überwiegenden Teil der planktischen Copepoden der Kieler Bucht.

Das Untersuchungsmaterial wurde mit Apstein-Planktonnetzen an den Stationen Boknis-Eck, am nördlichen Ausgang der Eckernförder Bucht und an der BellevueBrücke im Kieler Innenhafen im April-Mai 1972 gefangen. Die Copepoden wurden bis zur Weiterverarbeitung bei annähernd gleicher Wassertemperatur wie am Fangort gehältert. Nach einer Defäkationszeit von einem Tag wurden die Tiere einzeln unter dem Binokular in Reagenzgläser überführt, die mit ca. $10 \mathrm{ml}$ filtriertem Meerwasser gefüllt waren. Als Nahrung stand in ausreichendem Maße eine Kultur aus Chaetoceros decipiens, Chaetoceros socialis, Detonula cystifera, Skeletonema costatum und zwei Flagellatenarten zur Verfügung, die nicht näher bestimmt wurden.

Die Copepoden wurden bei der jeweils zur Fangzeit herrschenden Wassertemperatur $\left(7-8^{\circ} \mathrm{C}\right)$ im Dunkeln drei bis vier Tage gehältert. Die Nahrungspartikel wurden in Suspension gehalten. Anschließend wurden die Kulturen mit Formalin abgetötet und die ausgeschiedenen Faeces bei 300facher Vergrößerung mit Hilfe eines Meßokulars vermessen.

Da die Form und Größe der Kotballen nur durch die umgebende Chitin-Membran bestimmt wird, wurde nur die Länge und Breite dieser Hülle gemessen, nicht die des Inhalts, der die Hülle nicht in allen Fällen voll ausfüllt.

Die Hülle kann in einigen Fällen einen dünnen, schwanzförmigen Fortsatz bilden. Bei der Messung der Länge wurde deshalb zwischen „Länge mit Fortsatz" (LmF) und "Länge ohne Fortsatz“ (LoF) unterschieden.

Unter Berücksichtigung der jeweils annähernden Grundform der Faeces (zylindrisch oder ellipsoid) wurde das Volumen jedes untersuchten Kotballens nach den Formeln zur Volumenberechnung eines Zylinders und eines Ellipsoids bestimmt. Für jede untersuchte Copepodenart wurden für beide Geschlechter getrennt Mittelwert und Standardabweichung ermittelt. Die dünnen Fortsätze der Kotballen wurden bei der Volumenberechnung nicht berücksichtigt, da ihr Volumen verhältnismäßig sehr gering war und sie nie Kotmaterial enthielten.

Die experimentell gefundenen Werte wurden mit Hilfe des Chi-Quadrat-Tests auf signifikante Abweichungen von der Normalverteilung getestet. In über $95 \%$ aller Fälle war eine solche Abweichung nicht gegeben. Bei den weiteren statistischen Berechnungen wurde davon ausgegangen, daß in allen Fällen eine Normalverteilung vorlag. 
Die Unterschiede der Mittelwerte der einzelnen Parameter wurden mit Hilfe des t-Tests abgesichert. Da in den vorliegenden Fällen die Varianzen und die Anzahl der Stichproben der einzelnen Parameter ungleich waren, wurde die für diesen Fall zu wählende Sonderform der Formel zur Berechnung von "t" und der zur Verfügung stehenden Freiheitsgrade angewandt (SACHS, 1973; p. 214). Als Signifikanzgrenze wurde in allen Fällen das 5-\%-Niveau gewählt. Die Rechenarbeiten wurden mit Hilfe eines Olivetti-Programma-101- und eines Wang-720-B-Tischrechners ausgefuhrt.

\section{ERGEBNISSE}

Untersucht wurden insgesamt 1041 Kotballen von 55 Tieren. Die Grundform der Faeces war bei der Art Oithona similis annähernd ein Ellipsoid. Bei den Arten Acatia bifilosa, Centropages hamatus und Psendocalanus elongatus war die Form angenähert zylindrisch.

Bei einem Vergleich der Mittelwerte der Länge und Breite der Kotballen der vier Copepodenarten (Tab. 1) mit Hilfe des t-Tests ergab sich, daß sich die Faeces der verschiedenen Arten in mindestens einem der untersuchten Parameter signifikant unterschieden. Bei den Arten Acartia bifilosa und Centropages bamatus bestand ein solcher Unterschied ebenfalls zwischen den Korballen der beiden Geschlechter.

Tabelle 1

Größenmerkmale der Faeces von vier Copepodenarten der Kieler Bucht. Mittelwerte mit Standardabweidungen. $\mathrm{a}=$ Anzahl der untersuchten Tiere, $\mathrm{b}=$ Anzahl der untersuchten Kotballen, $\mathrm{c}=\mathrm{LoF}$ (Länge ohne Fortsatz) der Kotballen $(u \mathrm{~m}), \mathrm{d}=\mathrm{LmF}$ (Länge mit Fortsatz) der Kotballen $(\mu \mathrm{m}), \mathrm{e}=$ Breite der Kotballen $(\mu \mathrm{m}), \mathrm{f}=$ Volumen der Kotballen $\left(\mu \mathrm{m} \times 10^{-3}\right)$

\begin{tabular}{|c|c|c|c|c|c|c|}
\hline Species & a & $\mathrm{b}$ & $\mathrm{c}$ & $\mathrm{d}$ & $\mathrm{e}$ & $f$ \\
\hline $\begin{array}{l}\text { Acartia } \\
\text { bifilosa } 90 \\
\text { Acartia }\end{array}$ & 14 & 192 & $142 \pm 54$ & $143 \pm 53$ & $53 \pm 17$ & $348 \pm 258$ \\
\hline $\begin{array}{l}\text { bifilosa o o } \\
\text { Centropages }\end{array}$ & 4 & 132 & $123 \pm 45$ & $125 \pm 44$ & $34 \pm 4$ & $110 \pm 56$ \\
\hline $\begin{array}{l}\text { bamatus } 99 \\
\text { Centropages }\end{array}$ & 12 & 156 & $139 \pm 50$ & $157 \pm 50$ & $43 \pm 10$ & $226 \pm 167$ \\
\hline bamatus of 8 & 7 & 79 & $110 \pm 44$ & $135 \pm 49$ & $33 \pm 7$ & $110 \pm 90$ \\
\hline $\begin{array}{l}\text { similis } \text { ofo } \\
\text { Oithona }\end{array}$ & 16 & 181 & $36 \pm 9$ & $36 \pm 9$ & $26 \pm 6$ & $13 \pm$ \\
\hline $\begin{array}{l}\text { similis of } \\
\text { Pseudocalanus }\end{array}$ & 4 & 62 & $36 \pm 7$ & $36 \pm 7$ & $26 \pm 5$ & $13 \pm 6$ \\
\hline $\begin{array}{l}\text { elongatus } \\
\text { Psendocalanus }\end{array}$ & 7 & 106 & $157 \pm 51$ & $164 \pm 52$ & $31 \pm 6$ & $123 \pm 70$ \\
\hline elongatus 80 & 6 & 133 & $157 \pm 54$ & $163 \pm 54$ & $31 \pm 10$ & $129 \pm 94$ \\
\hline
\end{tabular}

Die Aussagen über die Charakteristika der Faeces der vier Copepodenarten beziehen sich auf Ergebnisse, die bei Kulturexperimenten gewonnen wurden, also unter erheblicher Veränderung der natürlichen Umwelt der Tiere. Um zu untersuchen, inwieweit die gefundenen Merkmale mit denen von Kotballen übereinstimmen, die von 
Copepoden im freien Wasser produziert wurden, wurde im April-Mai 1972 bei Boknis-Eck eine Sinkstoffalle (ZEITZschel, 1965) ausgebracht. Das hiermit aufgefangene sedimentierte Seston wurde unter dem Mikroskop auf Faeces untersucht.

Es wurden 163 Kotballen vermessen. Als Unterscheidungsmerkmal diente die Länge der Kotballen ohne Fortsatz (Abb. 1). Deutlich zeigt sich ein Maximum des Vorkommens im Größenbereich von 30-40 $\mu \mathrm{m}$, der Klasse, in der auch die durchschnittliche Länge der Faeces der Art Oithona similis liegt. Auch die Grundform der

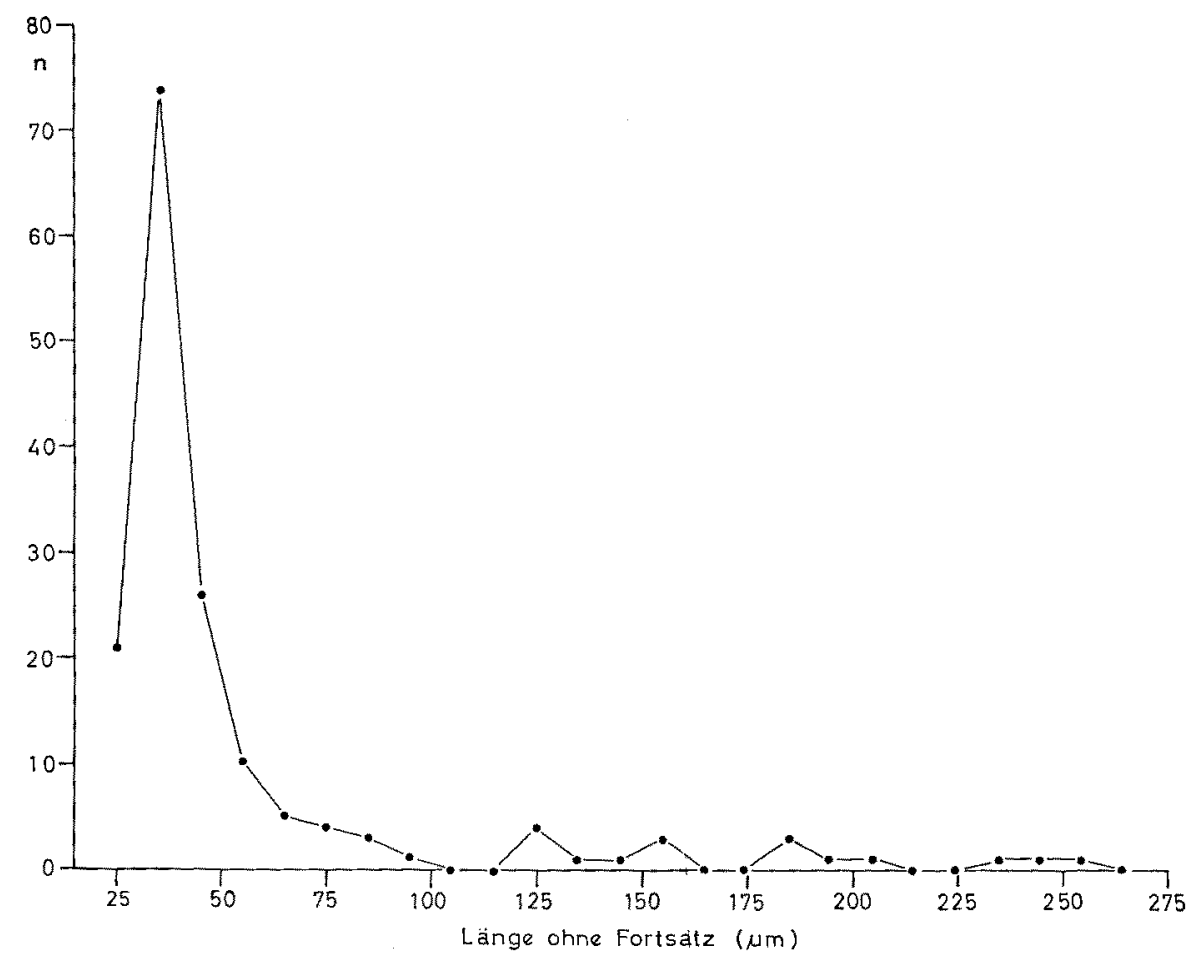

Abb. 1: Länge ohne Fortsatz (LoF) der in sedimentiertem Seston gefundenen Faeces planktischer Copepoden. Anzahl der Kotballen (n) pro Längenklasse (Klassenbreite $10 \mu \mathrm{m}$ )

Kotballen entspricht bis zu einer Größe von $60 \mu \mathrm{m}$ angenähert einem Ellipsoid, der im Experiment für die Faeces von Oithona similis bestimmten Form. Das arithmetische Mittel der Länge dieser in der Sinkstoffalle gefundenen ellipsoiden Kotballen beträgt $\overline{\mathrm{x}}=37 \mu \mathrm{m}$ bei einer Standardabweichung von $\mathrm{s}=8 \mu \mathrm{m}$. Dies stimmt recht gut mit dem experimentell für Faeces von Oithona similis bestimmten Wert überein $(\overline{\mathrm{x}}=$ $36 \mu \mathrm{m} ; \mathrm{s}=8 \mu \mathrm{m}$ ).

Das Längenmittel der zylindrischen Kotballen liegt bei $\bar{x}=141 \mu \mathrm{m}(\mathrm{s}=72 \mu \mathrm{m})$. Dieser Wert entspricht in etwa dem Mittelwert der LoF der Faeces von Acartia biflosa, Pseudocalanus elongatus und Centropages hamatus $(\overline{\mathrm{x}}=138 \mu \mathrm{m})$.

Auffällig ist die große Anzahl der Kotballen von Oithona similis in den Sinkstoffen, da diese Art zur Zeit der Probennahme der Sinkstoffe lediglich $24 \%$ des 
Bestandes der planktischen Copepoden bildete, während Pseudocalanus elongatus, Acartia bifilosa und Centropages hamatus zusammen $76 \%$ ausmachten. Dies würde für eine sehr viel höhere Produktionsrate der Art Oithona similis an Faeces sprechen. Tatsächlich befanden sich in den Kleinkulturen dieser Art jeweils etwa zehnmal mehr Kotballen pro Tier als in denen der anderen Arten.

\section{DISKUSSION}

Die Möglichkeit der artspezifischen Zuordnung der Kotballen zu verschiedenen Copepodenarten ermöglicht es, durch Analyse sedimentierten Sestons Aussagen über Vorgänge innerhalb der Wassersäule zu machen. Zwar berichten RAymoNT \& GRoss (1942), daß eine Anderung im Nahrungsangebot eine Anderung in Form und Größe der Faeces bei Calanus finmarchicus zur Folge hatte. Es handelte sich jedoch im wesentlichen um eine Änderung in der Ausbildung des schwanzförmigen Fortsatzes, der bei den hier vorliegenden Untersuchungen nie mit Kotmaterial gefüllt war. Der Hauptteil der Kotballen und somit die Menge der ausgeschiedenen Substanz blieb, insbesondere bei den Männchen, in Form und Größe relativ konstant. Betrachtet man die große Übereinstimmung in Form und Größe der Faeces aus den hier vorliegenden Kulturexperimenten mit denen aus den Freilanduntersuchungen, so kann auch in diesem Fall von einer großen Konstanz gesprochen werden.

Eine Analyse sedimentierter Kotballen erlaubt also Aussagen über die Zusammensetzung der Copepodenpopulation des betreffenden Zeitraumes. Eine Bestimmung der Anzahl der Copepoden ist dabei nur beschränkt möglich, da die Produktionsrate an Faeces z. B. bei Calanus finmarcbicus bei den Weibchen zwischen 25 und 119 pro Tier und Tag variieren kann (RAYMONT \& GRoss, 1942) und zudem stark von der Wassertemperatur abhängig ist (MARSHALL \& ORR, 1955). Eine grobe Abschätzung der Copepodenproduktion in der Wassersäule innerhalb eines bestimmten Zeitraumes setzt also zusätzliche Kenntnisse über die herrschenden Umweltfaktoren voraus. Ohne diese Kenntnisse ist lediglich eine Aussage über die qualitative Zusammensetzung der Copepodenpopulation möglich.

\section{ZUSAMMENFASSUNG}

1. Die Kotballen der planktischen Copepodenarten Acartia bifilosa, Centropages hamatus, Oithona similis und Pseudocalanus elongatus werden in ihrer Form, Länge und Breite beschrieben.

2. Die aus Länge, Breite und Grundform der Faeces berechneten durchschnittlichen Volumina liegen zwischen $13 \times 10^{3} \mu \mathrm{m}^{3}$ für beide Geschlechter der Art Oithona similis und $348 \times 10^{3} \mu \mathrm{m}^{3}$ für die Kotballen der Weibchen der Art Acartia bifilosa.

3. Die Dimensionen der Faeces der vier untersuchten Arten zeigen in mindestens einem der untersuchten Parameter signifikante Unterschiede. Im Falle der Arten Acartia bifilosa und Centropages hamatus sind auch die Größen der Kotballen beider Geschlechter signifikant verschieden. 
4. Ein Vergleich von Labor- und Freilanduntersuchungen zeigt, daß die in sedimentiertem Seston gefundenen Faeces planktischer Copepoden sich mit Hilfe der experimentell gefundenen Charakteristika den dominierenden Arten zuordnen lassen.

Anmerkung: Die Arbeit entstand im Rahmen des Sonderforschungsbereichs 95 „Wechselwirkung Meer-Meeresboden" der Universität Kiel (Publikationsnummer 6). Sie stellt die gekürzte Form der Diplomarbeit des Verfassers dar.

\section{ZITIERTE LITERATUR}

Forster, G. R., 1953. Peritrophic membranes in the Caridea (Crust. Decap.). J. mar. biol, Ass, U.K. 32, 315-318.

Gould, D. T., 1957. A peritrophic membrane in calanoid copepods. Nature, Lond. 179, 325-326.

Hillebrandt, M., 1972. Untersuchungen über die qualitative und quantitative Zusammensetzung des Zooplanktons in der Kieler Bucht während der Jahre 1966-1968. Diss., Univ. Kiel, 138 pp.

Johannes, R. E. \& Satom, M., 1966. Composition and nutritive value of fecal pellets of a marine crustacean. Limnol. Oceanogr. 11, 191-197.

Marshall, S. M. \& OrR, A. P., 1955. On the biology of Calanus finmarchicus. VIII. Food uptake, assimilation and excretion in adult and stage V Calanus. J. mar. biol. Ass. U.K. 34, 495-529.

Moore, H. B., 1931. The muds of the Clyde Sea Area III. Chemical and physical conditions; rate and nature of sedimentation and fauna. J. mar. biol. Ass. U.K. 18, 325-358.

Newell, R., 1965. The role of detritus in the nutrition of two marine deposit feeders, the prosobranch Hydrobia ulvae and the bivalve Macoma baltbica. Proc, zool. Soc. Iond. 144, $25-45$.

Osterberg, C., Carex, A. G. \& Curl, J., 1963. Acceleration of sinking rate of radionuclides in the ocean. Nature, Lond. 200, 1276-1277.

Raymont, J. E. G. \& Gross, F., 1942. On the feeding and breeding of Calanus finmarcbicus under laboratory conditions. Proc. R. Soc. Edinburgh 61, 267-287.

SACHS, L., 1973. Angewandte Statistik. Springer, Berlin, 544 pp.

Schrader, H. J., 1971. Fecal pellets: role in sedimentation of pelagic diatoms. Science, N. Y. $174,55-57$.

SMAYDA, T. J., 1969. Some measurements on the sinking rate of fecal pellets. Limnol. Oceanogr. 14, 621-626.

Zerrzschel, B., 1965. Zur Sedimentation von Seston, eine produktionsbiologische Untersuchung von Sinkstoffen und Sedimenten der westlichen und mittleren Ostsee. Kieler Meeresforsch. 21, 55-80.

Anschrift des Autors: Dr. P. Martens

Biologische Anstalt Helgoland (Litoralstation)

D-2282 List

Bundesrepublik Deutschland 